\title{
Degenerative Lumbar Spondylolisthesis with Spinal Stenosis: A Comparative Study of 5-Year Outcomes Following Decompression with Fusion and Microendoscopic Decompression
}

\author{
Takato Aihara ${ }^{1}$, Tomoaki Toyone ${ }^{2}$, Yasuaki Murata ${ }^{3}$, Kazuhide Inage ${ }^{4}$, Makoto Urushibara ${ }^{1}$, Juntaro Ouchi ${ }^{1}$
}

\author{
${ }^{1}$ Department of Orthopaedic Surgery, Funabashi Orthopedic Hospital, Funabashi, Japan \\ ${ }^{2}$ Department of Orthopaedic Surgery, School of Medicine, Showa University, Tokyo, Japan \\ ${ }^{3}$ Department of Orthopaedic Surgery, Teikyo University Chiba Medical Center, Ichihara, Japan \\ ${ }^{4}$ Department of Orthopedic Surgery, Graduate School of Medicine, Chiba University, Chiba, Japan
}

Study Design: Retrospective review of prospectively collected outcome data.

Purpose: To compare 5-year outcomes following decompression with fusion (FU) and microendoscopic decompression (MED) in patients with degenerative lumbar spondylolisthesis (DLS) and to define surgical indication limitations regarding the use of MED for this condition.

Overview of Literature: There have been no comparative studies on mid- or long-term outcomes following FU and MED for patients with DLS.

Methods: Forty-one consecutive patients with DLS were surgically treated. Sixteen patients first underwent FU (FU group), and 25 then underwent MED (MED group). The 5-year clinical outcomes following the two surgical methods were compared using the Japanese Orthopaedic Association Back Pain Evaluation Questionnaire.

Results: The degree of improvement (DOI) for social life function was significantly greater in the MED group than in the FU group. Although not statistically significant, DOIs for the other four functional scores were also greater in the MED group than in the FU group. However, patients with a large percentage of slippage in the neutral position might experience limited improvement in low back pain, those with a large percentage of slippage at maximal extension might experience limited improvement in three functional scores, and those with a small intervertebral angle at maximal flexion might have limited improvement in three functional scores after MED for DLS. Therefore, we statistically compared the DOls between the FU and MED groups regarding the preoperative percentage of slippage in the neutral position among patients with greater than $20 \%$ slippage, the preoperative percentage of slippage at maximal extension among patients with greater than $15 \%$ slippage, and the intervertebral angle at flexion among patients with angles lesser than $-5^{\circ}$; however, there were no statistically significant differences between the two groups.

Conclusions: MED is a useful minimally invasive surgical procedure that possibly offers better clinical outcomes than FU for DLS.

Keywords: Degenerative lumbar spondylolisthesis; 5-Year outcome; Comparative study; Decompression with fusion; Microendoscopic decompression

Received Mar 25, 2017; Revised Apr 29, 2017; Accepted Jun 8, 2017

Corresponding author: Takato Aihara

Department of Orthopaedic Surgery, Funabashi Orthopedic Hospital, 1-833 Hazama, Funabashi-city, Chiba 274-0822, Japan

Tel: +81-47-425-5585, Fax: +81-47-425-6592, E-mail: aihara@fff.or.jp 


\section{Introduction}

From the results of a systematic review to identify whether there is an advantage to instrumented or noninstrumented spinal fusion over decompression alone for patients with degenerative lumbar spondylolisthesis (DLS), a satisfactory clinical outcome was considered significantly more likely with fusion than with decompression alone [1]. Aihara et al. [2] compared outcomes between patients undergoing decompression with fusion (FU) and microendoscopic decompression (MED). Their short-term study suggested that MED was a useful minimally invasive surgical procedure that provides better clinical outcomes than FU for DLS, although a high slippage percentage in the neutral position could lead to inadequate improvement in low back pain after MED. To our knowledge, no previous study compared mid- or long-term outcomes following FU and MED for patients with DLS. The present study was performed to compare 5-year outcomes between FU and MED among patients with DLS and to define surgical indication limitations concerning the use of MED for this condition.

\section{Materials and Methods}

\section{Patient selection and surgical procedures}

Between May 2005 and August 2007, 43 consecutive patients, diagnosed with degenerative spondylolisthesis and spinal stenosis, met the criteria for inclusion. Their symptoms had been unresponsive to nonoperative treatment during a sufficient trial period. They had single or double levels of DLS based on plain radiographs and imaging studies consisting of a myelogram and contrast-enhanced computed tomography and magnetic resonance imaging, which revealed spinal stenosis at the level of spondylolisthesis. Patients who previously underwent spine surgery were ineligible. The first 17 patients were treated with FU (FU group), while MED was performed in the subsequent 26 patients (MED group). One patient each in the FU and MED groups were lost to follow-up within 5 years after their initial operations, and they were excluded. In the FU group, 8 patients with segmental kyphosis underwent decompressive laminectomy and posterior lumbar interbody fusion with pedicle screws and interbody cages for segmental kyphosis correction, seven patients without segmental kyphosis underwent decompressive laminec- tomy and posterolateral fusion using pedicle screws, and one patient underwent slippage-reducing L4-L5 anterior interbody fusion. MED, which is an endoscopic procedure performed using a tubular retractor (METRx; Medtronic Sofamor Danek, Memphis, TN, USA), was used to achieve sufficient decompression while preserving the posterior structures of the spine. Sixteen patients in the MED group presented with unilateral neurological symptoms, and a unilateral approach was used for unilateral decompression. Nine patients in the MED group exhibited bilateral neurological symptoms, and a unilateral approach was used for bilateral decompression [3]. All 41 operations were performed by the same surgeon.

\section{Patients}

The FU group included 10 women and six men, and the MED group included 11 women and 14 men. Gender differences between the groups were not significant. The ages of patients in the FU and MED groups were not significantly different according to an unpaired $t$-test (Table 1). The levels of slippage in the FU group were L4 and both L3 and L4 in 13 and three patients, respectively, and those in the MED group were L4 in 17 patients, L3 in three patients, L5 in one patient, and both L3 and L4 in four patients. The study protocol was approved by the Institutional Review Board (IRB approval no., 20110606).

\section{Imaging}

Plain radiographs of the lumbosacral spine (including anteroposterior, standing lateral [neutral], and standing flexion-extension lateral) of all patients were taken prior to surgery to measure the percentage of slippage at the slip level as well as the intervertebral angle (in degrees) between the adjacent vertebral end plates at the operative level according to the lateral flexion-extension radiographs $[2,4,5]$. A standing lateral (neutral) radiograph was used to measure the percentage of slippage using the method of Taillard [2,6-8]. Vertebral slippage exceeding $5 \%$ was considered to indicate DLS $[2,8]$. Percentage translation during flexion-extension and the intervertebral range of motion on the basis of maximal flexion and extension radiographs were also calculated. All preoperative radiological measurements were statistically compared by an unpaired $t$-test, and no significant differences were found between the two groups (Table 2). 
Table 1. Patient data $(n=41)$

\begin{tabular}{lccc} 
Variable & FU group $(\mathrm{n}=16)$ & MED group $(\mathrm{n}=25)$ & $p$-value \\
Age at surgery (yr) & $64.3 \pm 8.98$ & $62.7 \pm 9.74$ & 0.602 \\
Operation time (min) & $152 \pm 45.4$ & $87.5 \pm 26.7$ & $<0.05$ \\
\hline Blood loss at surgery (g) & $547 \pm 306$ & $87.8 \pm 68.8$ & $<0.05$ \\
Postoperative hospitalization (day) & $16.6 \pm 7.18$ & $9.40 \pm 8.63$ & $<0.05$ \\
\hline
\end{tabular}

Values are presented as mean \pm standard deviation.

FU, decompression with fusion; MED, microendoscopic decompression.

Table 2. Preoperative radiographic results of 48 operated levels

\begin{tabular}{lccc} 
Variable & FU group (n=19) & MED group (n=29) & $p$-value \\
\hline Slipping in neutral position (\%) & $14.8 \pm 6.88$ & $13.3 \pm 5.54$ & 0.418 \\
\hline Slipping at flexion (\%) & $18.4 \pm 7.99$ & $16.7 \pm 6.70$ & 0.428 \\
\hline Slipping at extension (\%) & $13.0 \pm 8.40$ & $10.8 \pm 5.38$ & 0.330 \\
\hline Translation during flexion-extension (\%) & $5.47 \pm 5.68$ & $5.92 \pm 4.18$ & 0.757 \\
\hline Intervertebral angle at flexion $\left({ }^{\circ}\right)$ & $-3.32 \pm 5.89$ & $-2.09 \pm 5.56$ & 0.467 \\
Intervertebral angle at extension $\left({ }^{\circ}\right)$ & $7.13 \pm 6.92$ & $8.55 \pm 4.85$ & 0.443 \\
\hline Intervertebral range of motion $\left({ }^{\circ}\right)$ & $10.4 \pm 4.83$ & $10.6 \pm 5.18$ & 0.899 \\
\hline
\end{tabular}

Values are presented as mean \pm standard deviation.

FU, decompression with fusion; MED, microendoscopic decompression.

\section{Outcome assessment}

Clinical 5-year outcomes were evaluated using the Japanese Orthopaedic Association Back Pain Evaluation Questionnaire (JOABPEQ) [9], which consists of five functional scores selected from the Roland Morris Disability Questionnaire and short form-36. The functional scores represent low back pain, lumbar function, walking ability, social life function, and mental health. The JOABPEQ formula, which is used to establish a measurement scale for each functional score, was adjusted so that the minimum and maximum functional scores were 0 and 100, respectively, with higher scores reflecting a better condition. The degree of improvement (DOI) was calculated as postoperative score-preoperative score. Five patients required repeat operations within 5 years after their initial surgeries, and their clinical outcomes were evaluated immediately before these second operations.

\section{Statistical analysis}

There is no generally accepted definition of instability [10]. Therefore, to determine the surgical indication limitations regarding the use of MED for DLS, 20 single
(L3/4 or L4/5) levels of slippage in the MED group were classified into two groups based on all preoperative radiological measurements (percentage of slippage in the neutral position, percentage of slippage and intervertebral angle at maximal flexion and extension, and percentage translation during flexion-extension and intervertebral range of motion) [2]: a group including measurements of the 10 largest levels (group L) and a group including measurements of the 10 smallest levels (group S). Data from five patients were discarded, including four patients with double levels of degenerative spondylolisthesis and one patient with L5 degenerative spondylolisthesis, with L5/S1 motion that differed from L3/4 and L4/5 motion [2]. All DOIs of groups L and S were statistically compared via an unpaired $t$-test.

Operative time, blood loss amount at surgery, postoperative hospitalization duration, and all DOIs in the FU and MED groups were statistically compared via an unpaired $t$-test. Pre- and postoperative JOABPEQ scores between the two groups were statistically compared using the Mann-Whitney $U$-test. For all analyses, a $p$-value of $<0.05$ was considered statistically significant [9]. 
Table 3. Preoperative Japanese Orthopaedic Association Back Pain Evaluation Questionnaire scores

\begin{tabular}{lccc} 
Variable & $\begin{array}{c}\text { FU group } \\
(\mathrm{n}=16)\end{array}$ & $\begin{array}{c}\text { MED group } \\
(\mathrm{n}=25)\end{array}$ & $p$-value \\
Low back pain & $38.4 \pm 33.3$ & $46.3 \pm 33.8$ & 0.449 \\
Lumbar function & $57.8 \pm 34.8$ & $54.3 \pm 29.5$ & 0.677 \\
Walking ability & $21.9 \pm 20.9$ & $23.4 \pm 21.2$ & 0.786 \\
Social life function & $37.3 \pm 21.2$ & $25.7 \pm 18.0$ & 0.0754 \\
Mental health & $42.6 \pm 21.9$ & $38.8 \pm 17.2$ & 0.407 \\
\hline
\end{tabular}

Values are presented as mean \pm standard deviation.

FU, decompression with fusion; MED, microendoscopic decompression.

Table 4. Postoperative Japanese Orthopaedic Association Back Pain Evaluation Questionnaire scores

\begin{tabular}{lccc} 
Variable & $\begin{array}{c}\text { FU group } \\
(\mathrm{n}=16)\end{array}$ & $\begin{array}{c}\text { MED group } \\
(\mathrm{n}=25)\end{array}$ & -value \\
\hline Low back pain & $68.8 \pm 33.5$ & $83.4 \pm 30.2$ & 0.0936 \\
Lumbar function & $74.0 \pm 26.9$ & $80.7 \pm 25.3$ & 0.293 \\
Walking ability & $62.1 \pm 38.0$ & $75.4 \pm 29.5$ & 0.237 \\
Social life function & $64.9 \pm 29.7$ & $75.7 \pm 24.3$ & 0.305 \\
Mental health & $58.0 \pm 18.8$ & $60.2 \pm 18.2$ & 0.862 \\
\hline
\end{tabular}

Values are presented as mean \pm standard deviation.

FU, decompression with fusion; MED, microendoscopic decompression.

\section{Results}

The operative time $(p<0.001)$, blood loss amount at surgery $(p<0.001)$, and postoperative hospitalization duration ( $p=0.00630)$ were significantly smaller in the MED group than in the FU group (Table 1). The preoperative score for social life function was lower in the MED group, but no significant differences were observed between the two groups in terms of preoperative scores for low back pain, lumbar function, walking ability, and mental health (Table 3). The postoperative score for low back pain was higher in the MED group, but no significant differences were observed between the two groups concerning the postoperative scores for lumbar function, walking ability, social life function, and mental health (Table 4). The DOI for social life function was significantly greater in the MED group than in the FU group $(p=0.0310)$. In addition, although not statistically significant, the DOIs for low back pain, lumbar function, walking ability, and mental health were
Table 5. Degrees of improvement in FU and MED groups

\begin{tabular}{lccc} 
Variable & $\begin{array}{c}\text { FU group } \\
(\mathrm{n}=16)\end{array}$ & $\begin{array}{c}\text { MED group } \\
(\mathrm{n}=25)\end{array}$ & $p$-value \\
Low back pain & $32.4 \pm 40.6$ & $42.2 \pm 40.8$ & 0.476 \\
\hline Lumbar function & $18.5 \pm 35.5$ & $27.4 \pm 37.6$ & 0.474 \\
\hline Walking ability & $40.2 \pm 33.8$ & $52.0 \pm 36.0$ & 0.300 \\
\hline Social life function & $27.5 \pm 30.1$ & $49.9 \pm 32.0$ & $<0.05$ \\
\hline Mental health & $15.4 \pm 28.4$ & $21.3 \pm 26.0$ & 0.498 \\
\hline
\end{tabular}

Values are presented as mean \pm standard deviation.

Degree of improvement was calculated by the following formula: postoperative score-preoperative score.

FU, decompression with fusion; MED, microendoscopic decompression.

greater in the MED group (Table 5).

The intraoperative complication of cerebrospinal fluid leakage in two patients in the MED group was controlled with a fibrin sealant with no additional treatment required. Postoperative transient foot drop was observed in one patient in the FU group, and postoperative transient urinary retention was observed in one patient in the MED group. The clinical outcomes of the two patients were not affected by these complications. Postoperative pseudarthrosis was observed in two patients in the FU group; specifically, one patient had persistent low back pain requiring postoperative pain management, and this complication eventually affecting the clinical outcome, whereas the other patient remained symptom-free and clinical outcome remained unaffected. Major internal complications such as pulmonary, cardiac, and cerebrovascular morbidity were not observed; surgical complications such as nerve injury and surgical site infection were also not observed.

Two patients in the FU group required repeat operation. In one patient, posterior migration of the interbody cages required their removal via an extraperitoneal approach, with L4-L5 anterior interbody fusion being performed 11 months after L4-L5 decompressive laminectomy and posterior lumbar interbody fusion. The other patient with low back pain because of adjacent degenerative disease required L2-L3 posterior decompression and transforaminal lumbar interbody fusion using pedicle screws 51 months after L3-L5 decompressive laminectomy and posterior lumbar interbody fusion.

Three patients in the MED group required repeat operation. The first underwent hemilaminotomy 7 months after bilateral L3/4 posterior decompression via a unilateral 


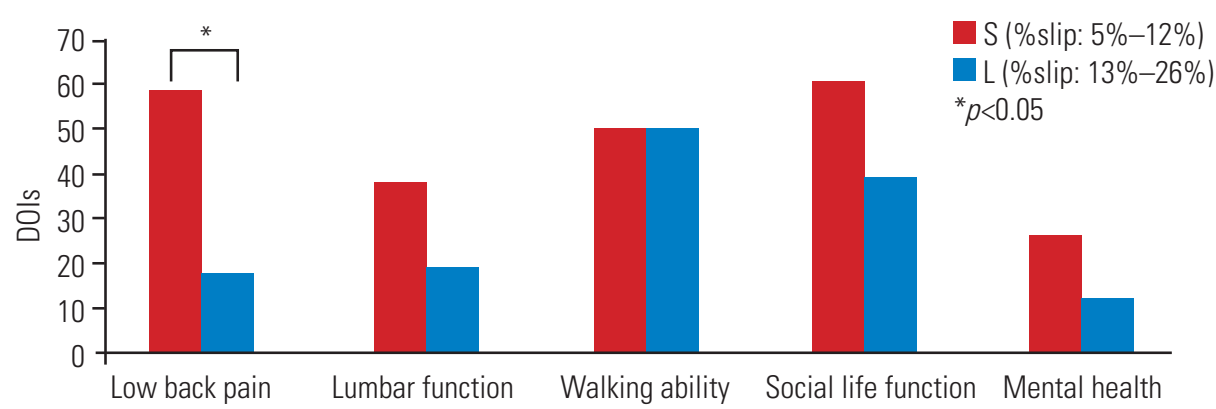

Fig. 1. Mean degrees of improvement (DOIs) in the preoperative percentage of slippage in the neutral position in groups $L$ and $S$.

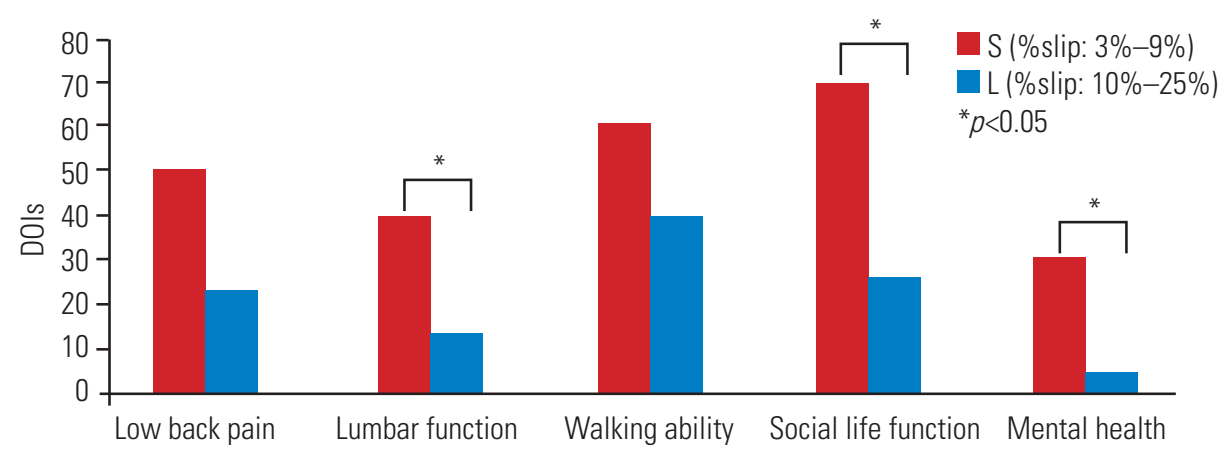

Fig. 2. Mean degrees of improvement (DOIs) in the preoperative percentage of slippage at maximal extension in groups $L$ and $S$.

approach because of left leg numbness. The second underwent posterior decompression and L4-L5 posterolateral fusion using pedicle screws due to low back pain and right sole numbness 8 months after bilateral L3/4 and L4/5 posterior decompression via a unilateral approach. The third underwent L4-L5 transforaminal lumbar interbody fusion with pedicle screws because of bilateral leg numbness 47 months after bilateral L4/5 posterior decompression via a unilateral approach. These symptoms may have been related, at least in part, to residual instability and increasing olisthesis postoperatively.

In terms of DOIs regarding the percentages of slippage at maximal flexion and intervertebral angles at maximal extension as well as percentage translation during flexion-extension and intervertebral ranges of motion, there were no significant differences between groups $\mathrm{L}$ and $\mathrm{S}$. The DOI for low back pain was significantly greater in group $S$ than in group L concerning the percentage of slippage in the neutral position ( $p=0.00362$ ) (Fig. 1); the DOIs for lumbar function, social life function, and mental health were significantly greater in group $\mathrm{S}$ regarding the percentage of slippage at maximal extension $(p=0.00270$, $p<0.001$, and $p=0.0102$, respectively) (Fig. 2). Further, concerning the intervertebral angle at maximal flexion, the DOIs for lumbar function, walking ability, and social life function were significantly greater in group $\mathrm{L}$ than in group $S$ ( $p=0.0270, p=0.0358$, and $p=0.0358$, respectively) (Fig. 3). Therefore, the DOIs in the FU and MED groups in terms of the preoperative percentage of slippage in the neutral position among patients with more than $20 \%$ slippage, the preoperative percentage of slippage at maximal extension among those with greater than 15\% slippage, and the intervertebral angle at flexion among patients with angles lesser than $-5^{\circ}$ were statistically compared. Although statistically not significant, DOIs in all five functional scores were greater in the FU group than in the MED group in terms of preoperative percentage of slippage in the neutral position among the cases exceeding $20 \%$ slippage, and the preoperative percentage of slippage at maximal extension among those exceeding 15\% slippage. Although statistically not significant, DOIs in all five functional scores were greater in the MED group than in the FU group regarding the intervertebral angle at flexion among the cases lower than $-5^{\circ}$. 


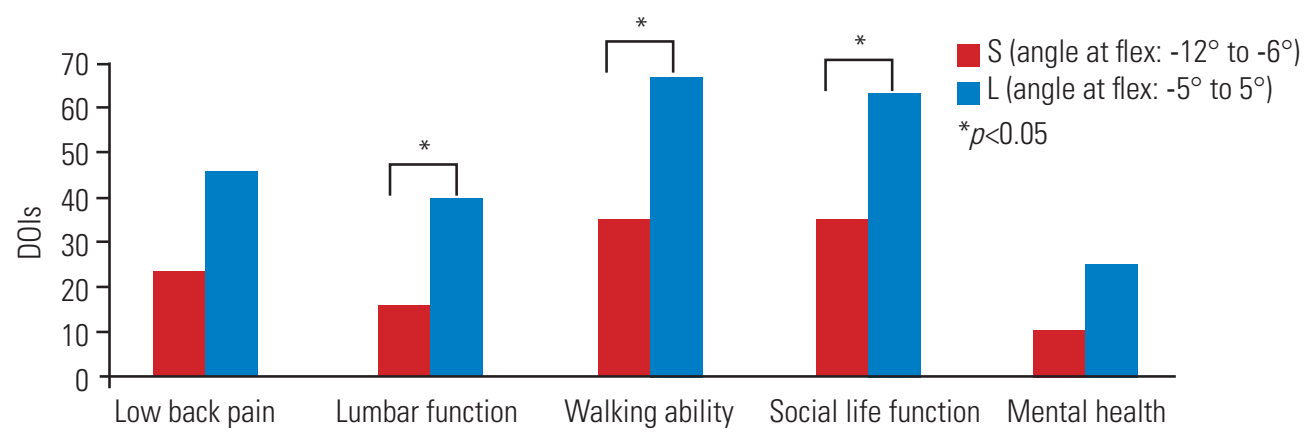

Fig. 3. Mean degrees of improvement (DOIs) in the preoperative intervertebral angle at maximal flexion (flex) in groups $\mathrm{L}$ and $\mathrm{S}$.

\section{Discussion}

Based on a study by Herkowitz and Kurz [4], decompressive laminectomy alone provided satisfactory results in $60 \%-96 \%$ of patients with DLS. Residual or recurrent pain in the back or lower limbs or both has been reported, but up to $73 \%$ of such patients more frequently complained of residual low back pain. Residual instability or increasing postoperative olisthesis may be related to such symptoms. In certain trials, olisthesis was found to worsen significantly more often in patients with poor outcomes [2]. By definition, degenerative spondylolisthesis means that the facet joints of a motion segment and supporting capsular ligaments are probably compromised. Decompressive laminectomy with partial excision of the facet joints then destabilizes the weakened segment, resulting in progressive olisthesis [4]. Thus, in line with these findings, until 2006, all our patients with spinal stenosis accompanying degenerative spondylolisthesis were treated using FU.

Since 2001, Ikuta et al. [3] have been treating most of their patients with spinal stenosis and DLS using microendoscopic posterior decompression. Notwithstanding the fact that the progression of spondylolisthesis and increased segmental sagittal motion after surgery occurred in 7 (19\%) of their patients, secondary fusion was only needed for one patient during follow-up. Using microendoscopic posterior decompression based on a unilateral approach for bilateral decompression that applies an endoscope at a $25^{\circ}$ angle, sufficient decompression, with preservation of the posterior structures of the spine, was obtained in almost all patients after surgery [2]. In addition, MED provides several other advantages over conventional decompression, such as reduced tissue trauma and wide, illuminated, and excellent visualization $[2,3]$. Based on these results, we have been using MED for all patients with spinal stenosis associated with degenerative spondylolisthesis since 2006, and the resulting data from the present study strongly suggest that MED is a useful minimally invasive surgical procedure that offers positive clinical outcomes for patients with DLS. The reason for this finding is that this procedure allows us to attain sufficient decompression while preserving the posterior structures of the spine.

However, certain limitations to the surgical indication for the application of MED for DLS should not be ignored. In the present study, 20 levels of slippage in patients in the MED group were classified into two groups according to the total preoperative radiological measurements: group L, with measurements of the 10 largest levels, and group S, with measurements of the 10 smallest levels. All DOIs were statistically compared between the two groups. According to the present results after MED for DLS, patients with large percentages of slippage $(13 \%-26 \%)$ in the neutral position might experience limited improvement in low back pain; those with large percentages of slippage (10\%-25\%) at maximal extension could experience limited improvement in lumbar function, social life function, and mental health; and those with small intervertebral angles $\left(-12^{\circ}\right.$ to $\left.-6^{\circ}\right)$ at maximal flexion might display limited improvement in lumbar function, walking ability, and social life function. Aihara et al. [2] recommended the use of MED in patients with DLS with less than 20\% slippage, but if slippage exceeds $20 \%$, posterior decompression and fusion with pedicle screws should be performed. In the current study, therefore, we statistically compared DOIs between the FU and MED groups concerning the preoperative percentage of slippage in the neutral position among patients with greater than $20 \%$ slippage, the preoperative percentage of slippage at maximal extension among patients with greater than $15 \%$ slippage, and the 
intervertebral angle at flexion among patients with angles lesser than $-5^{\circ}$; however, no statistically significant differences were noted between the two groups. Thus, taken together, considering the reduced invasiveness of MED and the 5-year outcomes, we believe that defining and establishing limitations of the surgical indications for the use of MED for DLS would be challenging.

There were some limitations to the current study. One was its small sample size, which made evaluation of the true validity of FU and MED associated with degenerative spondylolisthesis difficult. Further follow-up studies are required to accurately assess long-term outcomes. According to Martin et al. [1], numerous relevant randomized controlled trials and comparative observational studies were performed, but the conclusions from previous systematic reviews were limited by their reliance on scarcely available data from randomized controlled trials or dependence on data from pooled case series. We compared 16 earlier patients (FU group) who underwent FU with 25 later patients (MED group) who underwent MED. We believe that these two groups in the present study are comparable because of the absence of selection bias from any individual surgeon's interpretation of the patient's problems and choice of treatment [11]. Furthermore, the findings of the present study indicate that MED is a useful minimally invasive surgical procedure that promises to lead to better clinical outcomes than FU in patients with DLS. Nevertheless, it should be emphasized that, after MED, patients with high percentages of preoperative slippage in the neutral position might exhibit limited improvement in low back pain; those with high preoperative percentages of slippage at maximal extension might experience limited improvement in lumbar function, social life function, and mental health; and those with small preoperative intervertebral angles at maximal flexion might display limited improvement in lumbar function, walking ability, and social life function. We hope that the current findings can be viewed as representing preliminary data that can serve as an impetus for future randomized controlled trials.

\section{Conclusions}

MED is a useful minimally invasive surgical procedure that possibly offers better clinical outcomes than FU among patients with DLS.

\section{Conflict of Interest}

No potential conflict of interest relevant to this article was reported.

\section{References}

1. Martin CR, Gruszczynski AT, Braunsfurth HA, Fallatah SM, O'Neil J, Wai EK. The surgical management of degenerative lumbar spondylolisthesis: a systematic review. Spine (Phila Pa 1976) 2007;32:1791-8.

2. Aihara T, Toyone T, Aoki Y, et al. Surgical management of degenerative lumbar spondylolisthesis: a comparative study of outcomes following decompression with fusion and microendoscopic decompression. J Musculoskelet Res 2012;15:1250020.

3. Ikuta K, Tono O, Oga M. Clinical outcome of microendoscopic posterior decompression for spinal stenosis associated with degenerative spondylolisthesis: minimum 2-year outcome of 37 patients. Minim Invasive Neurosurg 2008;51:267-71.

4. Herkowitz HN, Kurz LT. Degenerative lumbar spondylolisthesis with spinal stenosis: a prospective study comparing decompression with decompression and intertransverse process arthrodesis. J Bone Joint Surg Am 1991;73:802-8.

5. Matsudaira K, Yamazaki T, Seichi A, et al. Spinal stenosis in grade I degenerative lumbar spondylolisthesis: a comparative study of outcomes following laminoplasty and laminectomy with instrumented spinal fusion. J Orthop Sci 2005;10:270-6.

6. Taillard W. Spondylolisthesis in children and adolescents. Acta Orthop Scand 1954;24:115-44.

7. Wiltse LL, Winter RB. Terminology and measurement of spondylolisthesis. J Bone Joint Surg Am 1983;65:768-72.

8. Kristof RA, Aliashkevich AF, Schuster M, Meyer B, Urbach H, Schramm J. Degenerative lumbar spondylolisthesis-induced radicular compression: nonfusion-related decompression in selected patients without hypermobility on flexion-extension radiographs. J Neurosurg 2002;97(3 Suppl):281-6.

9. Fukui M, Chiba K, Kawakami M, et al. JOA Back Pain Evaluation Questionnaire (JOABPEQ)/JOA Cervical Myelopathy Evaluation Questionnaire (JOACMEQ): the report on the development of revised versions April 16, 2007: the Subcommittee of 
the Clinical Outcome Committee of the Japanese Orthopaedic Association on Low Back Pain and Cervical Myelopathy Evaluation. J Orthop Sci 2009;14:34865.

10. Steiger F, Becker HJ, Standaert CJ, et al. Surgery in lumbar degenerative spondylolisthesis: indications, outcomes and complications: a systematic review.
Eur Spine J 2014;23:945-73.

11. Forsth P, Michaelsson K, Sanden B. Does fusion improve the outcome after decompressive surgery for lumbar spinal stenosis?: a two-year follow-up study involving 5390 patients. Bone Joint J 2013;95-B:9605. 